\title{
Interplay between disorder and quasi-regularity in CDW systems
}

\author{
I. BÂLDEA ${ }^{*}(1)$ and M. BǍDESCU ${ }^{* *}$ \\ *Universität Ulm, Abteilung für Mathematische Physik, Oberer Eselsberg, Postfach 4066, 89069 Ulm/Donau, \\ Germany \\ ${ }^{* *}$ Passive Component Design, IPEE Electroarges, $12-14$ Albesti Str, 0450 Curtea-de-Arges, Romania
}

\begin{abstract}
We develop here a microscopical model of quasi-regular impurity distribution driven by the CDW appropriate for highly mobile impurities or materials with fictitious CDW transition temperature. Thermal hysteresis effects at the CDW transition reported in the quasi-1D materials $\mathrm{NbSe}_{3}$ and $\mathrm{Ta}_{2} \mathrm{NiSe}_{7}$ could be interpreted in the framework of the present model. Possible connections with other (alloy, heavy-fermion) systems are also suggested.
\end{abstract}

\section{Introduction.}

The displacive motion of the impurities immersed into the one-dimensional system has been studied in detail previously as one kind of quasi-regularity driven by CDW [1]. As a further investigation of this problem we develop here a microscopical model for a different kind of quasi-regular impurity distribution driven by the CDW, consisting of the modulation in the probability of occupied sites. This modulation could be expected in two realistic cases. Firstly, this could happen in the case where impurities could migrate through the crystal, via atomic diffusion processes, towards those sites where the energy of their interaction with the CDW is minimum. Secondly, one may expect such a modulation in the distribution of impurities in connection with the manner in which a certain doped quasi-1D material is synthesised. If this preparation process yields a material whose stable state is a CDW one, one would expect that, in the case where the process is slow enough, impurities would have the possibility to drift towards those sites at which the minimization of the total energy occurs; thence, a modulation in the occupation probability with the same wave vector as the CDW. Such modulated impurity distributions have been previously postulated for KCP [2] and thiourea [3].

We present here a microscopic model which can describe the afore-mentioned modulation in the spatial distribution of impurities. The dependence on impurity concentration of the quantities characterizing the CDW state is obtained. Finally, we discuss the possible connection of our results with the experimental findings in several materials.

\section{Model of modulated distribution of impurities.}

In the case where the physical conditions permit, impurities could migrate through the crystal to reach those positions which are most favoured energetically in the presence of the Peierls-CDW distortion. 
In the corresponding state of thermodynamic equilibrium, the occupation probability depends on the energy of interaction between impurities and the host system. The energy $E\left(X_{n}\right)$ of interaction between an impurity located at $X_{n}$ and the CDW has the form:

$$
E\left(X_{n}\right)=-\rho_{1} U_{B} \cos \left(Q X_{n}+\varphi-\varphi_{B}\right)
$$

where $Q\left(=2 k_{F} \bmod G\right)$ is the CDW wave vector, $U_{B}$ and $\varphi_{B}$ are the amplitude and phase of the $Q$-Fourier component of electron-impurity scattering potential, respectively, and $\rho_{1}$ and $\varphi$ denote the amplitude and phase of CDW, respectively $\left(\rho(x)=\rho_{0}-\rho_{1} \cos (Q x+\varphi)\right)$. Once this interaction energy is known, the thermodynamics requires that the probability of occupying the position $X_{n}$ is $\left(\beta^{-1} \equiv k_{B} T\right)$ :

$$
w\left(X_{n}\right) \propto \exp \left[-\beta E\left(X_{n}\right)\right]
$$

Eq. (2) straightforwardly yields an extra $2 k_{F}$-potential $\Delta_{i}$ which acts in-phase with the contribution from the Peierls distorted lattice $\Delta_{L}$ on the electron gas. In the continuum limit, $\Delta_{i}$ has the expression:

$$
\Delta_{i}=f_{T} \frac{x}{d} U_{B} \frac{d}{d z} \log I_{0}(z)
$$

where $x$ is the fractional impurity concentration, $d$ is the intrachain lattice spacing, $z \equiv \beta \rho_{1} U_{B}$ and $I_{0}(z)$ is the modified Bessel function with index zero. Obviously, one expect realistically that only a (small) fraction from the total number of impurities is able to effectively reach (through atomic diffusion processes) the sites which are energetically most favoured (cf. Eq. (2)). This effect has been taken into account above by means of the parameter $f_{T}(\ll 1)$, presumably temperature dependent. To get the equation for the CDW order parameter $\Delta\left(=\Delta_{L}+\Delta_{i}\right.$ within the present approach $)$ one has to minimize the thermodynamic potential with the constraint provided by Eq. (3) because $\rho_{1}$ depends on $\Delta)$. Although the ensuing equation is rather complicated, analytic results can be obtained in the limits of low and high (i. e. near the critical) temperatures if one uses the behaviour of $I_{0}(z)$ for large and small values of the argument. For low temperatures $(\beta \Delta \gg 1)$, Eq. (3) leads to:

$$
\Delta_{i}=f_{0} \frac{x}{d} U_{B}
$$

which shows that in this limit the quantity $\Delta_{i}$ does not depend on $\Delta$. Actually, this model of $\Delta_{i}$ can also be used to describe a converse situation, where a quasi-regular impurity distribution drives the CDW or a system where the distortion has a different (e. g. chemical) origin or the material has a fictitious CDW transition temperature.

Close to the CDW-to-normal transition $(\beta \Delta \ll 1)$, one gets from Eq. (3):

$$
\Delta_{i}=f_{c} \frac{x}{d} \beta \rho_{1} U_{B}^{2}
$$

revealing that in this region the impurity quasi-regularity is driven by the CDW ( $\Delta_{i} \rightarrow 0$ as $\rho_{1} \rightarrow 0$ ).

\section{Results in the dilute limit.}

The full $x$-dependence of the quantities characterizing the CDW state will be presented elsewhere. Here we shall only give this dependence for the Peierls distortion amplitude $A_{L}$ at $T=0$ and the critical temperature $T_{c}$ in the dilute limit $(\gamma \simeq 1.78)$ :

$$
\begin{gathered}
\frac{T_{c}}{T_{c, 0}}-1=\left[\frac{2}{\gamma}\left(\frac{f_{c}}{\lambda^{2}}-\frac{\pi^{2}}{16}\right)-2 \frac{x_{c, 0}}{\lambda \alpha}\right] \frac{x}{x_{c, 0}} \equiv S_{T} x \\
\frac{A_{L}}{A_{L, 0}}-1=\left(x_{c, 0}+f_{0} \frac{1-\lambda}{\lambda} \frac{v_{F}}{U_{B}}-\frac{\pi}{8}-2 \frac{x_{c, 0}}{\lambda \alpha}\right) \frac{x}{x_{c, 0}} \equiv S_{A} x .
\end{gathered}
$$

Here $x_{c, 0}=v_{F} d \Delta_{0} / U_{B}^{2}$ is the critical concentration in the case of perfectly random impurity distribution and without including the elastic energy renormalization due to impurities [1], $\lambda$ denotes the electron-phonon coupling strength, $\alpha$ is a dimensionless quantity essentially proportional to the ratio of lattice-lattice and lattice-impurity elastic strengths; the quantities $f_{0}$ and $f_{c}$ denote the fractions of impurities which are quasi-regularly distributed at $T=0$ and $T=T_{c}$, respectively.

Depending on the values of parameters entering the model, even in the presently analysed dilute limit, one can get either a decrease or an increase in the quantities $T_{\mathrm{c}}$ and $A_{L}$ at increasing impurity content. This is illustrated in Fig. 1. 

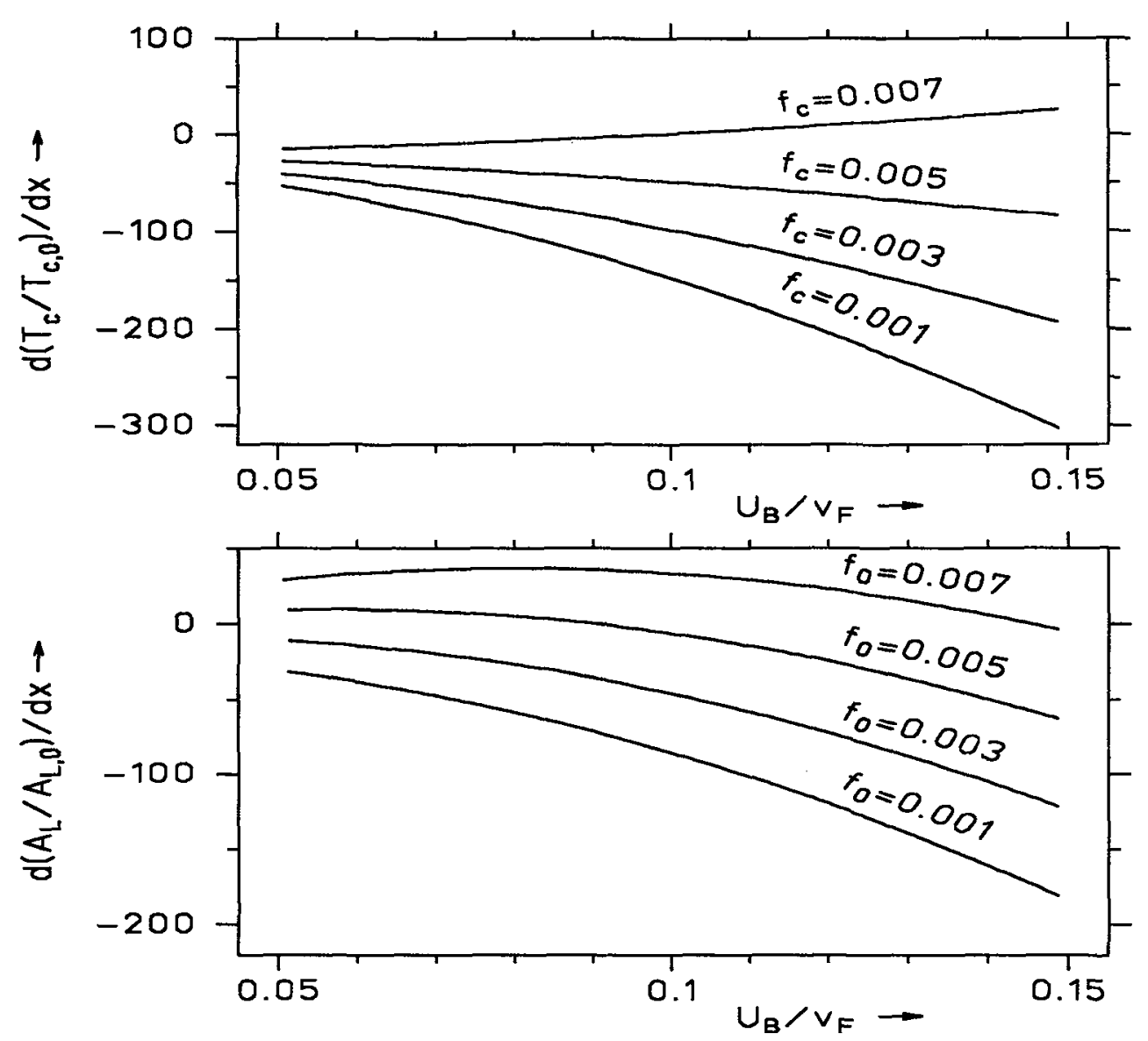

Figure 1: Dependence of the slopes $S_{T}=d\left(T_{c} / T_{c, 0}\right) / d x$ and $S_{A}=d\left(A_{L} / A_{L, 0}\right) / d x$ on the scattering parameter $U_{B} / v_{F}$ and the impurity fractions $\left(f_{0}\right.$ and $\left.f_{c}\right)$ (Eqs. $\left.(6,7)\right)$. The values of the remaining input parameters are: $\alpha=1,2 W d / v_{F}=1(W-$ electron bandwidth) and $\lambda=0.1$. The positive values of the slopes show that $T_{c}$ and $A_{L}$ can increase with $x$ for certain input parameter values.

\section{Thermal hysteresis at the CDW transition.}

As shown by Eqs. (6) and (7), the quasi-regularity in the impurity distribution rises the values of these quantities as compared to the case of perfect randomness (specified below by the subscript $r$ ):

$$
\begin{gathered}
\frac{T_{c}}{T_{c, r}}-1=\frac{2}{\gamma} \frac{f_{c}}{\lambda^{2}} \frac{x}{x_{c, 0}}, \\
\frac{A_{L}}{A_{L, 0}}-1=f_{0} \frac{1-\lambda}{\lambda} \frac{v_{F}}{U_{B}} \frac{x}{x_{c, 0}} .
\end{gathered}
$$

As discussed previously $[4,1]$, if one starts with a system in the normal state and cools it down, the CDW onset occurs at the critical temperature of the CDW transition with perfectly random impurity distribution $\left(T_{c, r}\right)$. Because this value is lower than the critical temperature of the CDW transition in the presence of quasi-regular-impurity distribution $T_{c}$ (cf. Eq. (8)) which furthermore enhances 
the CDW properties (e. g. $A_{L}$, cf. Eq. (9)), thermal hysteresis and first-order character at the CDW transition are expected (see $[4,1]$ for a more detailed discussion). On this basis, an explanation of the different values of the critical temperatures of the lower CDW transition (59K upon heating, $58 \mathrm{~K}$ upon cooling) and the (large) latent heats in $N b S e_{3}$ [5] can be suggested.

One may think that the thermal hysteretic behaviour observed in various CDW systems could be related to the relaxation of the CDW phase. Although the present status of both theoretical and experimental investigations cannot rule out an explanation which is different from that presently proposed, data reported in some other systems suggest that the at least in some cases the CDW phase should play but a small part in the thermal hysteretic behaviour. Perhaps most encouraging for the present interpretation is the thermal hysteresis found in the $2 k_{F}$-scattering intensities (i. e. directly related with a spatial modulated structure) in $\mathrm{Ta}_{2} \mathrm{NiSe}_{7}[6]$, along with the failure to detect a nonlinear electric conduction which is usually attributed to the depinned CDW phase mode. In addition, one should mention the phase transition of the heavy fermion system $U C u_{5}$ which removes parts of the Fermi surface, also displaying a thermal hysteresis [7].

The proposed modulation could also be related with the formation of the long-period superlattice structures from the disordered phase observed in $A g-M g$ alloys near $A g_{3} M g$ [8].

Although the afore-mentioned data could be invoked to give support to the hypothesis on impurity quasi-regularity, one should finally point out that all these experiments were not conduct primarily to maximize the effect we discussed here. To get the highest critical temperature $T_{c}$, samples in the normal state $\left(T \gg T_{c}\right)$ should be cooled down very slowly to temperatures $T \ll T_{c}$ (such that to allow impurities to follow the Peierls-CDW distortion), then quickly heated (in order to preserve as much impurity quasi-regularity as possible) up to the critical temperature $T_{c}$. Similarly, the lowest value of the critical temperature $T_{c, r}$ could be obtained by fast cooling the samples from temperatures $T \gg T_{c, r}$ to the transition region. A more detailed analysis of the various experimental data is deferred to a subsequent publication.

\section{Acknowledgements.}

One of the authors (I. B.) would like to thank the financial support provided by the Alexander von Humboldt Foundation during the main part of this work and by the German Academic Exchange Service (D A A D) in its initial stage as well as the hospitality extended to him at the University of Ulm. He also acknowledges with thanks valuable discussions with Profs. W. Wonneberger and G. Grüner.

\section{References}

[1] Bâldea I., Physica Scripta 42 (1990) 749 and references cited therein.

[2] Bâldea I. and Apostol M., Proc. $17^{\text {th }}$ Intern. Conf. on Low Temperature Physics, part 2, Ed. U. Eckern, A. Schmid, W. Weber and H. Wuhl (Elsevier Science B V, 1984) p. 1339; J. Phys. C: Solid State Physics 18 (1985) 6135.

[3] Lederer P., Montambaux G., and Jamet J. P., Molec. Cryst. Liquid Crystals 121 (1985) 99.

[4] Bâldea I., and Bădescu M., Physica Scripta 40 (1989) 311.

[5] Tomić S., Biljaković K., Djurek D., Cooper J. R., Monceau P. and Meerschaut A., Solid St. Commun. 38 (1981) 109.

[6] Fleming R. M., Sunshine S. A., Chen C. H., Schneemeyer L. F. and Waszczak J. V., Phys. Rev. B 42 (1990) 4954.

[7] Ott H. R., Rudigier H., Felder E., Fisk Z. and Batlogg B., Phys. Rev. Lett. 55 (1985) 1595.

[8] Fujino Y., Phys. Rev. B 42 (1990) 5373. 